\title{
Diphtheritic neuropathy, an analysis based on muscle and nerve biopsy and repeated neurophysiological and autonomic function tests
}

\author{
G SOLDERS, ${ }^{*} †$ I NENNESMO $\ddagger$ A PERSSON $†$ \\ From the Departments of Neurology,* Clinical Neurophysiology, $\dagger$ and Neuropathology, $\ddagger$ Huddinge University \\ Hospital, Sweden
}

SUMMARY A patient with diphtheritic neuropathy was investigated with repeated tests of parasympathetic and sympathetic vasomotor and sudomotor functions for one year after the onset of symptoms. Somatic nerve function was tested with nerve conduction studies and an index based on ten variables was used to follow the course of the neuropathy. Sural nerve and anterior tibial muscle biopsies were performed. A severe but shortlasting impairment of the parasympathetic vagal reflex arc was found. The recovery of this function paralleled the clinical course. Sympathetic functions were normal. The neurophysiological variables of somatic nerve function showed signs of a mainly demyelinating mixed sensory/motor neuropathy. The recovery of these variables was slow. The nerve and muscle biopsies demonstrated mild changes consistent with a mixed, demyelinating, noninflammatory neuropathy.

Diphtheritic neuropathy is relatively seldom seen today in the western world. Most reports are from the first half of this century. Since 1940 mortality from diphtheria (around $1-2 \%$ ), has been mainly attributed to diphtheritic heart disease. ${ }^{12}$ The incidence of peripheral neuropathy in diphtheria is estimated to be $20 \%{ }^{3}$ Demyelinating non-inflammatory neuropathy is seen with a delay of 8-12 weeks after the throat infection. ${ }^{3}$ There is nearly always a complete recovery of nerve function. ${ }^{12}$ The pathogenesis of the neuropathy is only partly understood but probably involves inhibition of the synthesis of myelin proteolipid and basic protein by diphtheritic toxin. ${ }^{4}$

Impairment of autonomic effector organ reflexes has been demonstrated in acute inflammatory neuropathy ${ }^{56}$ and in experimental allergic neuritis. ${ }^{7-9}$ To our knowledge there have been no previous reports of autonomic reflexes in diphtheritic neuropathy. We thought it useful to investigate the involvement of autonomic nerves during the course of the disease in a

Address for reprint requests: Göran Solders, MD, Department of Neurology, Huddinge Hospital, S-141 86 Huddinge, Sweden

Received 7 October 1988 and in revised form 12 February 1989. Accepted 17 February 1989 patient with diphtheritic neuropathy seen recently if our department.

\section{Case report}

The patient, a 61 year old man, had been operated on for a lumbar disc prolapse at the age of 38 but he was otherwise healthy. He was a smoker but had no history of alcohol abuse. In December 1984 he had a throat infection and the bacterial culture showed Corynebacterium diphtheriae non gravis. He was treated with 12000 IE diphtheriae immunoglobin intramuscularly and phenoxymethyl-penicillin orally. Erythromycin was later added. Thirteen days after the throat infection the patient experienced difficulty in swallowing and a paralysis of the soft palate was diagnosed. The dysphagia lasted for three weeks. There was no diplopia. Eight weeks after the throat infection ( $=$ week 0 in the recordings) the patient experienced numbness and paraesthesia in his hands and feet. After 9 weeks (= week 1) he had difficulty in walking and was referred to the department of neurology.

A moderate distal weakness was found with global areflexia and hypaesthesia for all modalities in the hands and below the knees. CSF examination showed a slightly raised $\mathrm{CSF} /$ plasma albumin ratio of 12.8 (normal $<10$ ) with no pleocytosis. There were no oligoclonal bands on isoelectric focusing. Routine blood examinations were normal.

The muscle weakness progressed and for two weeks the patient had to use a wheelchair. Twelve weeks after the throat 
infection there was a gradual improvement and after 16 weeks he was sent home. At follow up four months later he was almost free of symptoms.

\section{Methods}

Nerve conduction studies (ENeG) and autonomic function tests were carried out on eight occasions during weeks 3 to 45 after the onset of weakness.

ENeG was performed with conventional neurophysiological technique in the median, peroneal and sural nerves. Ten variables were recorded: motor conduction velocity (MCV) and distal latency (DL) in the median and peroneal nerves, sensory conduction velocity (SCV) and the amplitude of the nerve action potential (NAP) in the distal and proximal parts of the median nerve and in the sural nerve. Care was taken to make all recordings at normal skin temperature. The degree of neuropathy was expressed as an index, ENeG-Ix, calculated as the mean deviation (expressed in SD) of the ten variables from normal age-matched laboratory controls. The normal limit of the ENeG-Ix for individuals (ie for $p<0.05$ ) is $+2 \sqrt{\text { number of variables, }}$ thus in this study $\pm 0 \cdot 63 .^{10}$

Beat-to-beat variation of the electrocardiogram (R-R variation) was recorded and plotted on-line against time. The $R-R$ variation relative to mean $R-R$ interval was calculated during normal breathing (N\%) and during 1 minute of deep breathing at 6 breaths/min (D\%). Recording procedure and calculations have been described, in detail, earlier."

R-R interval, finger pulse amplitude (recorded by a photoplethysmograph) and galvanic skin response (GSR) were recorded during the Valsalva manoeuvre. The patient was asked to blow into a mouthpiece (with a small leak) and to maintain a pressure of $40 \mathrm{~mm} \mathrm{Hg}$ for $20 \mathrm{~s}$. The Valsalvaquotient (Valq) was calculated by dividing the longest $\mathbf{R}-\mathbf{R}$ interval after by the shortest interval during the manoeuvre. Reduction of finger pulse amplitude during Valsalva (Valp) was expressed as a percentage. GSR was recorded during a deep breath and during Valsalva and was graded as absent $(0)$, weak $(+)$, or strong $(++)$. Our normal values of these tests for a 61 year old man are $\mathrm{N} \%>8, \mathrm{D} \%>12$, Valq $>1 \cdot 17$, Valq $<40 \%$ and GSR + or ++ , respectively.

\section{Biopsies}

Muscle and nerve biopsies were performed 8 weeks after onset of weakness. The muscle biopsy was taken from the anterior tibial muscle using the percutaneous conchotome technique. The specimen was quickly frozen in isopenthane,
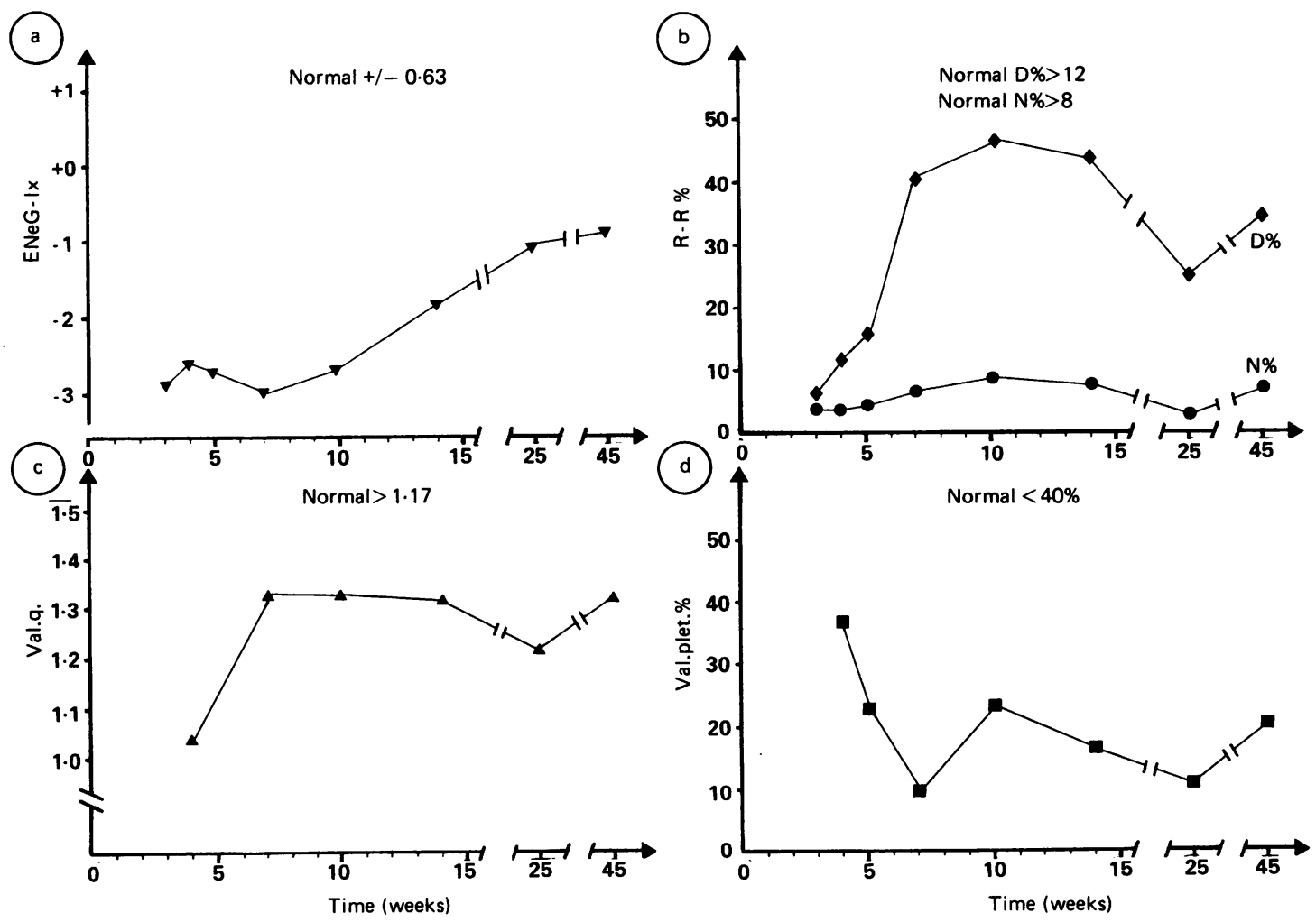

Fig 1 Neurophysiological recordings during weeks 3-45. (a) Electroneurography-Index (ENeG-Ix). (b) Heart beat variations $(R-R \%)$ during normal breathing $(N \%)$ and during deep breathing $(D \%)$. (c) Heart beat reaction to Valsalva (Valq). (d) Finger pulse amplitude reaction to Valsalva (Valp). 


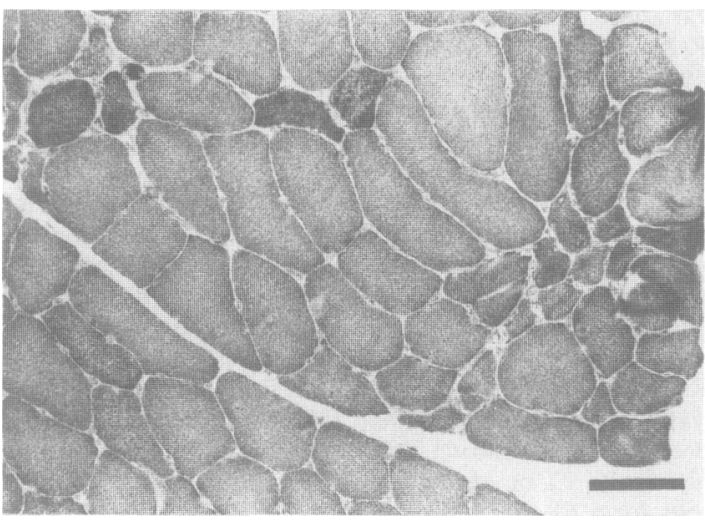

Fig 2 Biopsy from anterior tibial muscle (DPNH-reaction) demonstrating some scattered and a small group of atrophic angulated fibres (bar $=100 \mu \mathrm{m})$.

kept at its melting point by liquid nitrogen and cut at $-20^{\circ} \mathrm{C}$ in $6 \mu \mathrm{m}$ sections. The sections were stained with haematoxylin-eosin, Gomori trichrome, ATPase at $\mathrm{pH} 9.4$ with and

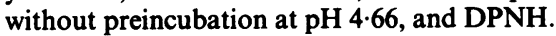

The sural nerve biopsy, performed under local anaesthesia, was taken from behind the lateral malleolus. Three fascicles were divided and a part of the specimen was fixed in $2.5 \%$ glutaraldehyde in $0.1 \mathrm{M}$ Sörensens phosphate buffer at $\mathrm{pH}$ 7.4 for 24 hours. Transversal and longitudinal sections were postfixed for 2 hours in $1 \%$ osmic acid, dehydrated and embedded in Lx-112 (Ladd Res, Vt) One $\mu$ m thick sections were cut and stained with toluidine blue. Ultrathin sections were stained with lead citrate and uranyl-acetate. One fascicle was dissected, osmificated and 25 fibres were teased. Some of the material was frozen and subjected to immunohistochemical staining for HLA-DR antigen and T-lymphocytes as described elsewhere. ${ }^{12}$ The rest of the material was embedded in paraffin, cut and stained with Luxol fast bluecresyl violet and haematoxylin-eosin.

Morphometrical evaluation was made on one $\mu \mathrm{m}$ thick transversal section using a semiautomatic technique (MOP Videoplan, Kontron, Munich). Fibre density (number of myelinated axons $/ \mathrm{mm}^{2}$ ) and the area of the myelinated fibres were measured. Based on the area, the circular diameter (that is, diameter of a circle with equal area) of the myelinated fibres were measured in 304 fibres.

\section{Results}

ENeG showed slowed conduction velocities, multiple conduction block and prolonged F-response latencies. The maximal impairment was seen around seven weeks after onset of weakness. After this, the neurophysiological tests showed a slow gradual improvement, but with pathological results even after one year. The change in ENeG-Ix over this period is shown in fig la.

The autonomic effector organ tests showed an early and prominent impairment of $R-R$ variations and Valsalva quotient that was maximal at 3-5 weeks after the onset of weakness. These tests showed a rapid recovery that paralleled the clinical improvement and at week 7 normal results were found (fig $1 \mathrm{~b}$ and $1 \mathrm{c}$ ). The Valp showed borderline results at week 3, but was normal at later testings (fig 1d). The GSR was normal during the whole test period.

In the muscle biopsy some scattered and occasional small groups of angulated atrophic fibres were seen (fig 2). The sural nerve showed a reduced number of myelinated nerve fibres (fibre density 5300 fibres/ $\mathrm{mm}^{2}$ ), (fig 3a). The fibre diameter histogram had a normal distribution of myelinated fibres with a mean diameter of $6.5 \mu \mathrm{m}$ (fig 4 ). In a few of the teased fibres
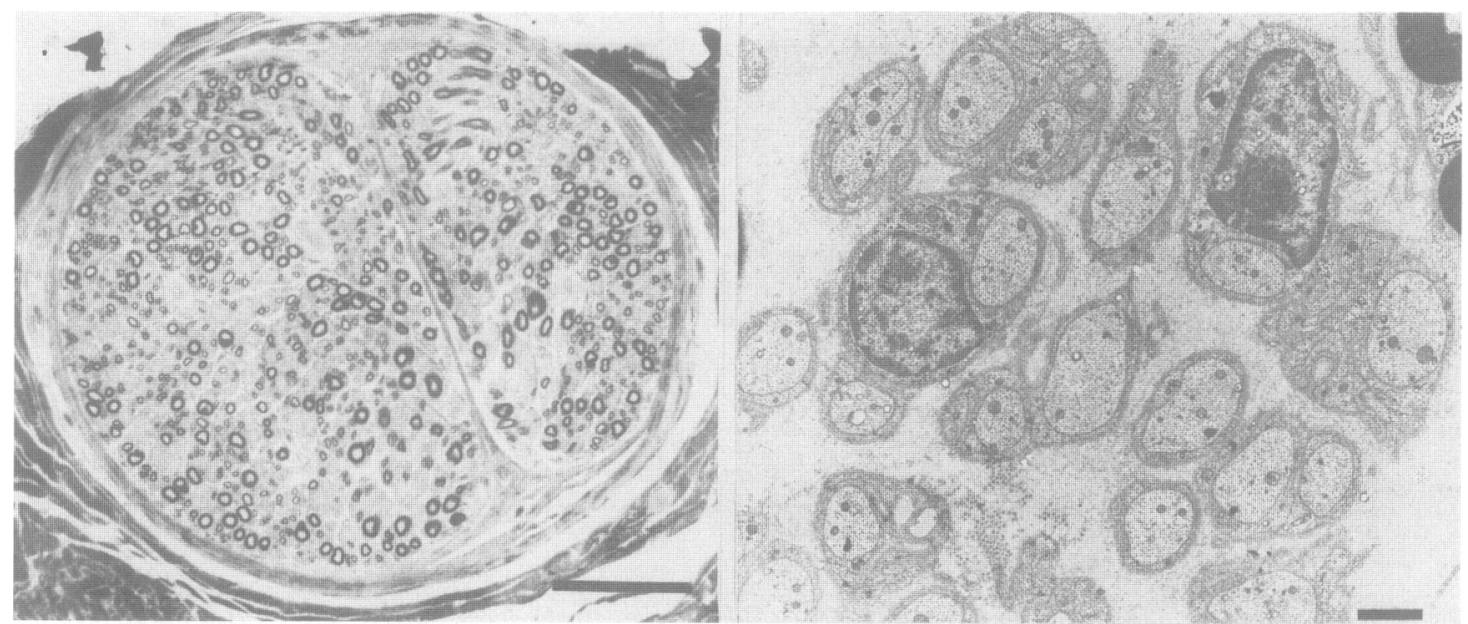

Fig 3 Transversal section of the sural nerve. (a) Light microscopy of a fascicle with reduced number of myelinated fibres (bar $=100 \mu \mathrm{m})$. (b) Electronmicroscopy showing a group of unmyelinated fibres of normal appearance (bar $=1 \mu \mathrm{m})$. 


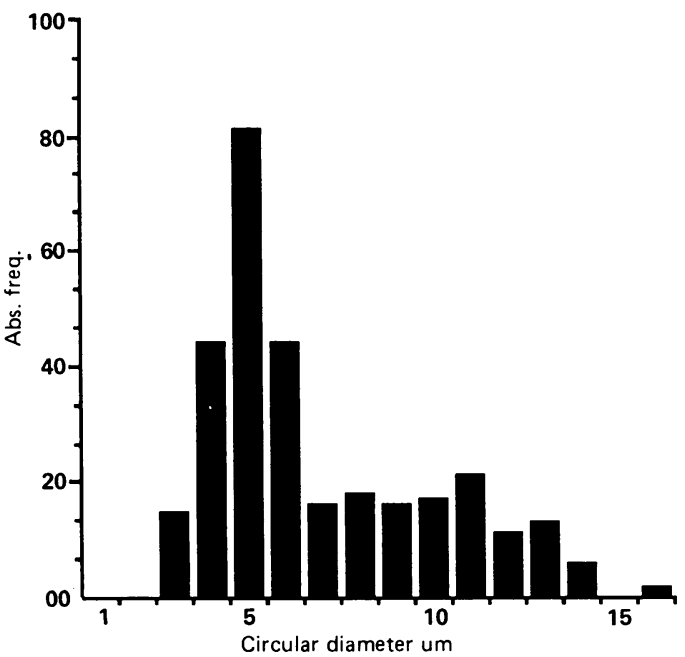

Fig 4 Histogram of the circular diameter of myelinated fibres showing a normal bimodal distribution.

there were signs of demyelination with short internodes and thin myelin sheaths. There were no inflammatory cell infiltrates. Monoclonal staining against HLA-DR antigen demonstrated expression on nonmyelinating Schwann cells and macrophages. Electron-microscopy showed unmyelinated fibres that were normal (fig $3 b$ ).

\section{Discussion}

This patient had typical clinical symptoms of diphthertic polyneurpathy with transient dysphagia 3-6 weeks after the throat infection and widespread sensory-motor deficits 8 weeks after the infection. ${ }^{1313}$

There was, as expected, an almost complete clinical recovery of the neuropathy in four months. The ENeG-Ix indicated a maximal impairment at weeks 3-10 and then a gradual improvement. However, it still remained below the normal level after one year. Similar results showing a slow return of conduction velocities, in spite of clinical improvement, have been reported in experimental diphtheritic neuropathy in guinea pigs. ${ }^{14}$ Slow conduction velocities in the acute phase of the disease have been reported. ${ }^{1516}$ In a follow-up study of 11 patients the maximal impairment was found during weeks 5-10 with normal conduction velocities in the median and peroneal nerves after 33 weeks. ${ }^{17}$ The ENeG-Ix used in our study is based on ten age adjusted parameters in the $\mathrm{ENeG}$ and is probably a more sensitive method than the conduction velocities alone for following the course of a neuropathy.

The autonomic effector organ tests revealed an early and prominent impairment in what are believed to be parasympathetic vagal functions, that is, the R-Rvariations and the heart reaction to the Valsalva manouevre. ${ }^{18}{ }^{19}$ Heart disturbances, especially myocarditis, is a common complication in diphtheria, ${ }^{21320}$ but to our knowledge there have been no previous reports on cardiac reflexes or other autonomic effector organ tests in diphtheritic neuropathy. The results of these tests followed the clinical recovery and $\mathrm{D} \%$ and Valq were normal 7 weeks after the onset of weakness. This and the slower return of the conduction velocities indicate great similarities to results in the GuillainBarré syndrome, ${ }^{521}$ where a demyelination and/or a conduction block in the thin myelinated vagal fibres have been postulated..$^{22}$

The fingerpulse-amplitude (Valp) and the GSR showed normal results throughout the study (except for a borderline low value of Valp at week 4) indicating normal function in the thin unmyelinated sympathetic vasomotor and sudomotor fibres.

Many thorough neuropathological studies have been made based on necropsy material from patients who died from diphtheritic paralysis. ${ }^{313}$ However, we have found no report on sural nerve biopsy findings from such cases. In necropsy material the pathological changes have been concentrated on the peripheral nervous system in the region of the dorsal root ganglia and adjacent parts of the dorsal, ventral, and mixed spinal nerve roots. In late cases, changes may occur more peripherally. Degeneration of the nodose ganglion $^{13}$ or the vagal nerve or other parts of the vegetative nervous system have been reported.' The typical changes have been patchy demyelination with thin and short internodes, but with preserved axonal continuity. In experimental diphtheritic neuropathy in the guinea-pigs there was a widening of the nodes of Ranvier before any symptoms occurred ${ }^{14}$ and in chickens similar early changes were found in the thin myelinated fibres with a widening of the internodal gap. $^{23}$

In our case, the sural nerve biopsy showed signs of demyelination with short internodes. The fibre density was slightly reduced and the fibre diameter histogram was normal. There was no infiltration of inflammatory cells in the biopsy specimen. The staining for HLADR antigen demonstrated expression of the antigen on non-myelinating Schwann cells and on macrophages.

Since the sural nerve contains only somatosensory and autonomic nerves, involvement of the motor nerves has to be deduced from assay of the muscle biopsy. The anterior tibial muscle showed signs of early neurogenic atrophy with scattered angular atrophic fibres. No inflammation was seen in the muscle. Similar results have been reported by Gibbels et al $^{24}$ in four cases, where neurogenic atrophy in type 2B muscle fibres and some targetoid fibres, were seen.

We have described a severe but relatively short 
lasting vagal autonomic dysfunction in a case with diphtheritic neuropathy. The recovery in the autonomic tests paralleled the clinical course whereas the nerve conduction studies on somatic nerves showed a more prolonged recovery.

Morphological changes have been demonstrated in the sural nerve and in the anteror tibial muscle that were consistent with a mixed sensory/motor demyelinating non-inflammatory neuropathy.

We thank Prof $\mathrm{K}$ Kristensson for his constructive criticism and $\mathrm{K}$ Kawa and $\mathrm{K}$ Lindqvist for their excellent technical assistance. The study was supported by research funds from the Karolinska Institute.

\section{References}

1 Scheid W. Diphtherial paralysis. An analysis of 2292 cases of diphtheria in adults which included 174 cases of polyneuritis. $J$ Nerv Ment Dis 1952;116:1095-101.

2 Hoel J, Holstberg A. Persistent diphtheritic heart disorders. Acta Med Scand 1953;145:393-405.

3 McDonald WI, Kocen RS. Diphtheritic neuropathy. In: Dyck PJ, Thomas PK, Lamert EH, Bunge R, eds. Peripheral Neuropathy vol II. Philadelphia: WB Saunders Co, 1984:2010-17.

4 Pleasure DE, Feldmann B, Prockop DJ. Diphtheria toxin inhibits the synthesis of myelin proteolipid and basic proteins by peripheral nerve in vitro. $J$ Neurochem 1973;20:81-90.

5 Persson A, Solders G. R-R variations in Guillain-Barré syndrome: a test of autonomic dysfunction. Acta Neurol Scand 1983;67:294-300.

6 Singh NK, Jaiswal S, Mista S, Srivastava PK. Assessment of autonomic dysfunction in Guillain-Barre syndrome and its prognostic implications. Acta Neurol Scand 1987;75:101-5.

7 Appenzeller O, Arnason BG, Adams RD. Experimental autonomic neuropathy: an immunologically induced disorder of reflex vasomotor function. J Neurol Neurosurg Psychiatry 1965;28:510-5.

8 Saksa M, Molnár GK, Riekkinen PJ. Bradycardic response to respiratory strain as a measure of autonomic dysfunction in experimental allergic neuritis. Acta Neurol Scand 1982;suppl
90:VIII:7.

9 Solders A, Persson A, Kristensson K, Hansson S. Autonomic dysfunction in experimental allergic neuritis. Acta Neurol Scand 1985;72:18-25.

10 Wennberg A. A neuropathy index based on motor-sensory electroneurography (ENeG). Acta Neurol Scand 1984;70: 107-11.

11 Persson A, Solders G. R-R variations, a test of autonomic dysfunction. Acta Neurol Scand 1983;67:285-93.

12 Schroder H, Olsson T, Solders G, Kristensson K, Link H. HLADR-expressing cells and T-lymphocytes in sural nerve biopsies. Muscle Nerve 1988;11:864-70.

13 Fisher CM, Adams RD. Diphtheritic polyneuritis, a pathological study. J Neuropathol Exp Neurol 1956;15:243-68.

14 Morgan-Hughes JA. Experimental diphtheritic neuropathy. A pathological and electrophysiological study. J Neurol Sci 1968;7:157-75.

15 Kazemi B, Tahernia C, Zandian K. Motor nerve conduction in diphtheria and diphtheritic myocarditis. Arch Neurol 1973;29:104-6.

16 Fasshauer K, Haupt W, Huffman G, Leven B. Elektrodiagnostische Befunde bei 5 Patienten mit Diphterie-Polyneuropathie. Nervenarzt 1977;48:391-6.

17 Kurdi A, Abdul-Kader M. Clinical and electrophysiological studies of diphtheritic neuritis in Jordan. $J$ Neurol Sci 1979;42:243-50.

18 Ewing DJ, Borsey DQ, Bellavere F, Clarke BF. Cardiac autonomic neuropathy in diabetes: Comparison of measures of $R-R$ interval variations. Diabetologia 1981;21:18-24.

19 Wieling W, van Brederode JFM, de Rijk LG, Borst C, Dunning AJ. Reflex control of heart rate in normal subjects in relation to age: a data base for cardiac vagal neuropathy. Diabetologia 1982;22:163-6.

20 Gore I. Myocardial changes in fatal diphtheria. A summary of $\bar{\gamma}$ observations in 221 cases. Am J Med Sci 1948;215:257-66.

21 Albers JW, Donofrio PD, Gonagle TK. Sequential electrodiagnostic abnormalities in acute inflammatory demyelinating polyradiculoneuropathy. Muscle Nerve 1985;8:528-39.

22 Tuck RR, McLeod JG. Autonomic dysfunction in Guillain-Barré $\supset$ syndrome. J Neurol Neurosurg Psychiatry 1981;44:983-90.

23 Cavanagh JB, Jacobs JM. Some quantitative aspects of diphtheritic neuropathy. Br J Exp Pathol 1964;45:309-22.

24 Gibbels E, Berger M, Herbolsheimer M, Korn A, Stammler A. Muskelbioptische Befunde bei der Diphterie-Polyneuropathie. Acta Neuropathol (Berl) 1981;55:307-18. 